\title{
Genetically Defined Oligodendroglioma Is Characterized by Indistinct Tumor Borders at MRI
}

\author{
(DD.R. Johnson, (DF.E. Diehn, (D) C. Giannini, (DR.B. Jenkins, DS.M. Jenkins, DI.F. Parney, and (D).J. Kaufmann
} O- EN

\begin{abstract}
BACKGROUND AND PURPOSE: In 2016, the World Health Organization revised the brain tumor classification, making IDH mutation and $1 p / 19 q$ codeletion the defining features of oligodendroglioma. To determine whether imaging characteristics previously associated with oligodendroglial tumors are still applicable, we evaluated the MR imaging features of genetically defined oligodendrogliomas.
\end{abstract}

MATERIALS AND METHODS: One hundred forty-eight adult patients with untreated World Health Organization grade II and III infiltrating gliomas with histologic oligodendroglial morphology, known 1p/19q status, and at least 1 preoperative MR imaging were retrospectively identified. The association of $1 \mathrm{p} / 19 \mathrm{q}$ codeletion with tumor imaging characteristics and ADC values was evaluated.

RESULTS: Ninety of $148(61 \%)$ patients had 1p/19q codeleted tumors, corresponding to genetically defined oligodendroglioma, and 58/148 (39\%) did not show 1p/19q codeletion, corresponding to astrocytic tumors. Eighty-three of 90 (92\%) genetically defined oligodendrogliomas had noncircumscribed borders, compared with 26/58 (45\%) non-1p/19q codeleted tumors with at least partial histologic oligodendroglial morphology $(P<.0001)$. Eighty-nine of 90 (99\%) oligodendrogliomas were heterogeneous on T1- and/or T2-weighted imaging. In patients with available ADC values, a lower mean ADC value predicted $1 \mathrm{p} / 19 \mathrm{q}$ codeletion $(P=.0005)$.

CONCLUSIONS: Imaging characteristics of World Health Organization 2016 genetically defined oligodendrogliomas differ from the previously considered characteristics of morphologically defined oligodendrogliomas. We found that genetically defined oligodendrogliomas were commonly poorly circumscribed and were almost always heterogeneous in signal intensity.

ABBREVIATIONS: IDH = isocitrate dehydrogenase; $\mathrm{WHO}=$ World Health Organization

$\mathbf{T}$ he classification and treatment of brain tumors, particularly oligodendroglial tumors, is rapidly evolving due to increasing awareness of the role of molecular factors. Several large clinical trials have demonstrated that overall survival is dramatically increased when patients with World Health Organization (WHO) grade II and III oligodendroglial tumors receive chemotherapy in addition to radiation therapy, particularly in pa-

Received August 24, 2016; accepted after revision November 10.

From the Departments of Radiology (D.R.J., F.E.D., T.J.K.), Pathology (C.G., R.B.J.), Health Sciences Research (S.M.J.), and Neurosurgery (I.F.P.), Mayo Clinic, Rochester, Minnesota.

Caterina Giannini and Robert B. Jenkins were partially funded by National Cancer Institute grant P50 CA108961.

Some aspects of the paper were previously presented at: Annual Meeting of the American Society of Neuroradiology and the Foundation of the ASNR Symposium, June, 4-9, 2011; Seattle, Washington.

Please address correspondence to Timothy J. Kaufmann, MD, Department of Radiology, Mayo Clinic, 200 First St SW, Rochester MN 55905; e-mail: Kaufmann.Timothy@mayo.edu

-- Indicates open access to non-subscribers at www.ajnr.org

三 Indicates article with supplemental on-line table.

http://dx.doi.org/10.3174/ajnr.A5070 tients with tumors having simultaneous whole arm deletions of $1 \mathrm{p}$ and $19 \mathrm{q} \cdot{ }^{1-3}$ This $1 \mathrm{p} / 19 \mathrm{q}$ codeletion had been previously recognized as a prognostic factor for better survival, ${ }^{4}$ but these studies provided proof that it was also predictive of response to therapy. $^{5}$

In 2016, the WHO released updated guidelines for brain tumor classification that made isocitrate dehydrogenase (IDH) mutation and 1p/19q codeletion the defining features of oligodendroglioma (oligodendroglioma $I D H$-mutant and $1 \mathrm{p} / 19 \mathrm{q}$ codeleted and anaplastic oligodendroglioma $I D H$-mutant and $1 \mathrm{p} / 19 \mathrm{q}$ codeleted). ${ }^{6}$ Whole arm $1 \mathrm{p} / 19 \mathrm{q}$ codeletion typical of oligodendroglioma occurs exclusively in isocitrate dehydrogenase gene mutation and telomerase reverse transcriptase (TERT) mutation. ${ }^{7}$ Grade II and III infiltrating gliomas without $1 \mathrm{p} / 19 \mathrm{q}$ codeletion are classified as astrocytomas and divided among $I D H$-mutant (most) and $I D H$-wild type (10\%-20\%). The designation "oligoastrocytoma not otherwise specified" is strongly discouraged, to be used primarily when molecular testing is not available.

A number of imaging features have long been considered characteristic, though not specific, to oligodendrogliomas: relatively 


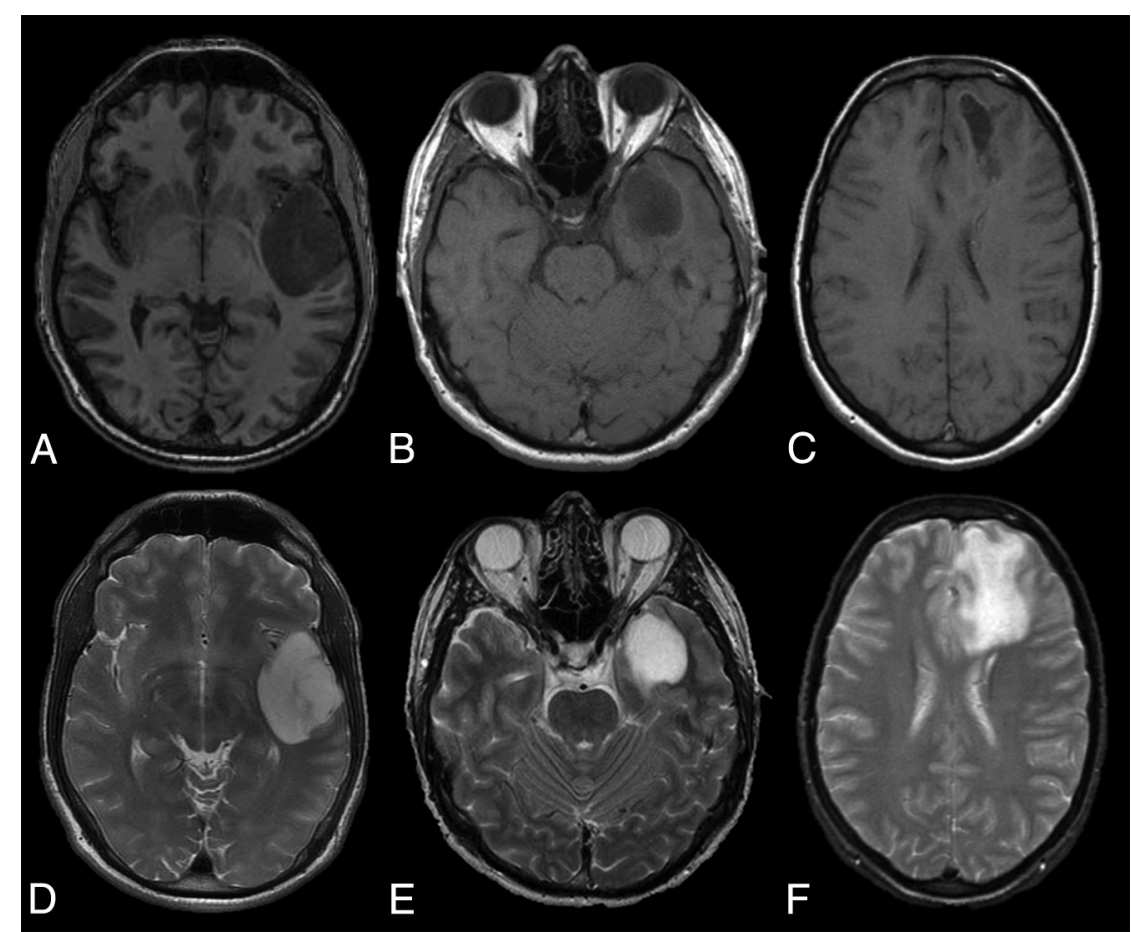

FIG 1. Examples of circumscribed ( $A$ and $D$ ), partially circumscribed ( $B$ and $E$ ), and noncircumscribed ( $C$ and $F$ ) tumor borders on axial T1-weighted (upper row) and T2-weighted (lower row) MR images. tumors that contained oligodendroglial elements at microscopy based on the 2007 WHO brain tumor classification system. ${ }^{9}$ Patients were excluded if the tumor was classified as an astrocytoma or a morphologic tumor subtype could not be assigned; if they did not have at least $1 \mathrm{MR}$ imaging study containing both T1- and T2-weighted images obtained before surgical intervention; or if they had received any chemo- or radiation therapy. Two board-certified neuroradiologists blinded to patient $1 \mathrm{p} / 19 \mathrm{q}$ status and histopathology evaluated preoperative MR images at a digital workstation (Advantage Workstation 4.3; GE Healthcare, Milwaukee, Wisconsin) and performed image analysis in consensus. The MR imaging examinations were performed at our institution and at outside institutions that had referred patients to us. Pulse sequences generally included pre- and postgadolinium T1- and T2-weighted imaging and T2-weighted FLAIR imaging. Other sequences, DWI, gradient-

sharply delineated margins, calcification, and internal cysts. ${ }^{8}$ These features must be re-evaluated following the WHO 2016 brain tumor update because those tumors previously classified as oligodendrogliomas or oligoastrocytomas but lacking $1 \mathrm{p} / 19 \mathrm{q}$ codeletion are now considered astrocytomas, and those tumors previously called oligoastrocytomas containing the $I D H$ mutation and $1 \mathrm{p} / 19 \mathrm{q}$ codeletion are now classified as oligodendrogliomas. Thus, it is not clear whether the features previously considered typical of oligodendroglioma are able to characterize genetically defined WHO 2016 oligodendrogliomas and distinguish them from astrocytomas.

We evaluated a large series of patients with morphologically defined oligodendroglial tumors (oligodendroglioma and oligoastrocytoma by WHO 2007 criteria) to determine whether MR imaging features could identify those patients with true genetically defined oligodendrogliomas (ie, those tumors with $I D H$ mutation and $1 \mathrm{p} / 19 \mathrm{q}$ codeletion). Because most patients with glioma undergo their initial imaging studies and often their initial surgical intervention in the community setting, we focused on MR imaging sequences that are routinely obtained as part of standard diagnostic MR imaging.

\section{MATERIALS AND METHODS}

\section{Patient Identification and Study Design}

Institutional review board approval with waived consent was obtained for this Health Insurance Portability and Accountability Act-compliant retrospective research study. Our institutional neuropathology data base was used to identify patients 18 years of age and older with WHO grade II or III infiltrating gliomas originally diagnosed as oligodendrogliomas and oligoastrocytomas whose tumors had undergone $1 \mathrm{p} / 19 \mathrm{q}$ fluorescent in situ hybridization testing to assess codeletion. This testing was performed on echo $\mathrm{T}^{*}$-weighted, and DSC perfusion imaging, were not consistently available.

Tumor location was assessed by the primary lobe of involvement and by whether the tumor burden was unilateral or bilateral (by crossing the midline or by multifocal disease). The following classifications were noted to reflect the predominant nature of each tumor: tumor margins completely circumscribed, partially circumscribed, or noncircumscribed (Fig 1); borders predominantly sharply interfaced with normal brain or indistinct (T1- and T2-weighted sequences) (Fig 2); contour predominantly smooth or irregular (Fig 2); and internal homogeneity versus nonhomogeneity (T1- and T2-weighted sequences). Circumscription of tumor margins was judged as a summary marker from all pulse sequences and was considered complete if $>75 \%$ of the tumor circumference was geographically marginated, as if one could trace a pencil line around the tumor. Partial circumscription was present if $50 \%-75 \%$ of the tumor circumference was circumscribed, and lack of circumscription was present if $<50 \%$ of the tumor circumference was circumscribed. The judgment of sharpversus-indistinct tumor borders was considered potentially related to circumscription and overlapping with it but more specifically looking at whether tumor interfaces with brain were sharp or blurred individually on both T1- and T2-weighted pulse sequences. Sharp-versus-indistinct border was chosen to reflect the strong predominance of the character of a given tumor.

The judgment of tumor contour as smooth or irregular was also considered potentially related to circumscription and tumor border sharpness but was specifically focused on whether tumor margins were convex outward as a predominant characteristic of a given tumor on T2-weighted images versus a mix of tumor margins convex outward and concave inward. Necrosis or cystic 


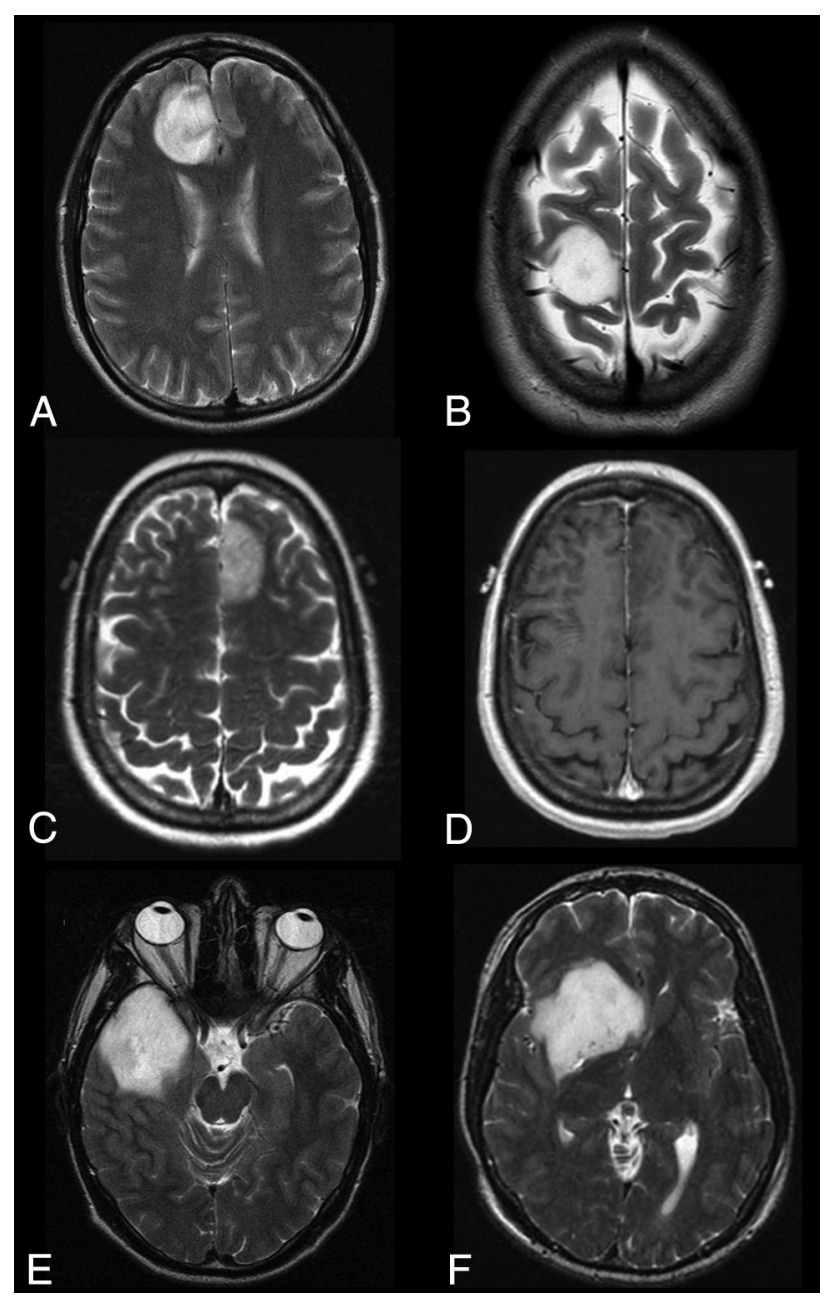

FIG 2. Examples of tumor classifications are as follows: circumscribed, with sharp smooth borders $(A)$; circumscribed, with sharp borders, but not smooth due to the extent of concave inward borders (B); circumscribed with sharp borders on T2-weighted image $(C)$ but not on T1-weighted image (D); partially circumscribed, with indistinct borders, but not smooth due to the extent of concave inward borders (E); and circumscribed, with predominantly sharp borders, but not smooth due to the extent of concave inward borders $(F)$. All images are T2-weighted, except $D$ as indicated.

change and the presence or absence of contrast enhancement were also determined. Paramagnetic susceptibility effect (or loss of signal, which might indicate hemorrhage or calcification) was evaluated with $\mathrm{T} 2^{\star}$-weighted imaging when available; otherwise, we assessed focal T1 shortening and/or focal signal loss on T2weighted images. When available, mean and median overall ADC was measured for each tumor from 2D ROIs. Given frequent glial tumor heterogeneity, we also sampled the highest and lowest $\mathrm{ADC}$ areas within each tumor by placing four $15-$ to $22-\mathrm{mm}^{2}$ ROIs in areas of visually low and high tumoral ADC, and the means of these low and high ADC ROIs were calculated, followed by measures of central tendency of those mean values across patients.

\section{Pathologic Correlation}

Tumor histopathologic diagnoses were obtained from pathology reports in the electronic medical record. Diagnoses were determined by board-certified neuropathologists by using the WHO
2007 update criteria. ${ }^{9}$ The $1 \mathrm{p} / 19 \mathrm{q}$ codeletion status, as determined by fluorescence in situ hybridization, was obtained from clinical records. IDH1- and IDH2- mutation analysis was available in a subset of cases; sequencing methods have been previously described. ${ }^{10}$ For this evaluation, the term "genetically defined oligodendroglioma" is used for pathologically proved WHO grade II and III infiltrating gliomas, which contain $1 \mathrm{p} / 19 \mathrm{q}$ codeletion by fluorescence in situ hybridization; and the term "astrocytoma" is used in the absence of $1 \mathrm{p} / 19 \mathrm{q}$ codeletion. From a technical standpoint, knowledge of $I D H$ mutation is necessary for complete classification, and this was only available in a subset of patients. However, $I D H$ mutation is always present in patients with $1 \mathrm{p} / 19 \mathrm{q}$ codeleted oligodendroglioma and is likely present in at least $80 \%$ of the noncodeleted tumors in this study, because the association between oligodendroglial morphology at microscopy and $I D H$ mutation is well-established.

\section{Statistical Analyses}

The associations of $1 \mathrm{p} / 19 \mathrm{q}$ codeletion status with the recorded imaging features, tumor type, and tumor grade were assessed. Data were compared between groups with $\chi^{2}$ tests or Fisher exact tests (when appropriate) for nominal variables and with the Kruskal-Wallis test for continuous or ordinal variables. Multivariable logistic regression models were used to assess the association of $1 \mathrm{p} / 19 \mathrm{q}$ codeletion (genetic oligodendroglioma classification) with imaging characteristics, and results were summarized with odds ratios, 95\% confidence intervals, and C statistics. All analyses were performed by using SAS, Version 9 (SAS Institute, Cary, North Carolina). $P$ values $\leq .05$ were considered statistically significant.

\section{RESULTS \\ Study Population}

One hundred eighty-five patients with previously untreated WHO grade II or III infiltrating gliomas who underwent tumor $1 p / 19 q$ codeletion testing at the time of the first operation were identified. We excluded patients with the following: morphologically pure astrocytic tumors $(n=26)$, age younger than 18 years at the time of diagnosis $(n=5)$, either T1- or T2-weighted MR imaging sequences unavailable $(n=2)$, research authorization declined $(n=2)$, tumor that could not be assigned a morphologic subtype $(n=1)$, and prior craniotomy at another institution $(n=$ $1)$. One hundred forty-eight patients with infiltrating gliomas with histologic oligodendroglial morphology met the criteria for inclusion. Of these, 90 of 148 (61\%) demonstrated 1p/19q codeletion and 58 of 148 (39\%) did not. Patient demographic characteristics are shown in Table 1 . MR images were predominantly $2 \mathrm{D}$ rather than $3 \mathrm{D}$ volumetric, and most scans were performed at $1.5 \mathrm{~T}$.

\section{Tumor Histology and IDH-Mutation Status}

Histologic tumor characteristics and $I D H$-mutation statuses are shown in Table 2. Thirty-six of 42 (86\%) tumors previously characterized as oligodendrogliomas were $1 \mathrm{p} / 19 \mathrm{q}$ codeleted, and 6 of $42(14 \%)$ were not. Of the 106 tumors previously classified as oligoastrocytomas by the 2007 criteria, $54(51 \%)$ were $1 \mathrm{p} / 19 \mathrm{q}$ codeleted and 52 were not, corresponding to astrocytoma, $I D H$ - 
Table 1: Patient demographics

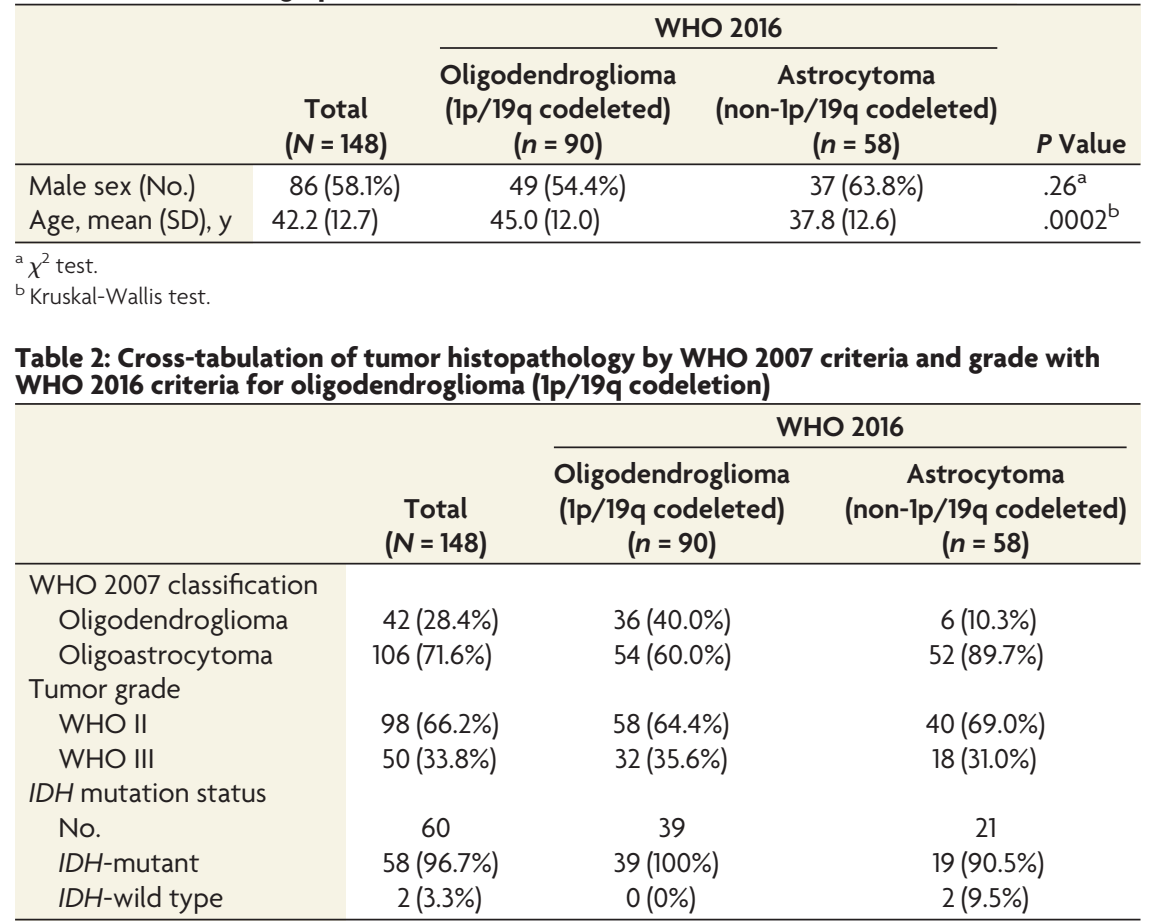

significant for each marker of border sharpness evaluated, compared with astrocytic tumors with some oligodendroglial morphology $(P<.0001$ for each). The small cohort of internally homogeneous tumors was very unlikely to be codeleted $(P=.003$ and 0.01 for T1- and T2-weighted images, respectively). Paramagnetic susceptibility, necrosis or cystic change, and contrast enhancement were relatively common in both oligodendrogliomas and astrocytomas, with no significant difference in prevalence. Figure 3 demonstrates prototypical genetically defined oligodendrogliomas and astrocytomas.

In the multivariable analysis (Table 3 ), tumor location ( $P$ values calculated, excluding 2 patients with cerebellar or deep gray location), lack of tumor circumscription $(P<.0001)$, and older age at diagnosis $(P=.002)$ were independently significant predictors of $1 p / 19 q$ codeleted oligodendroglioma. The odds of oligodendroglioma increased with

mutant, or IDH-wild type. As expected, 39 of 39 (100\%) codeleted tumors in which $I D H$-mutation sequencing was available had mutations of either IDH1 or IDH2, making them oligodendrogliomas by the WHO 2016 classification system. Among the tumors without $1 \mathrm{p} / 19 \mathrm{q}$ codeletion, of those 21 in which $I D H$ status was known, 19 (90\%) demonstrated mutation.

\section{Tumor Location}

The proportion of tumors that crossed the midline or displayed bilateral multifocal involvement was approximately $20 \%$ for both genetically defined oligodendrogliomas and astrocytomas. Oligodendrogliomas were most commonly centered in the frontal lobe (66/90, 73\%), followed by the parietal (15/90, 17\%) and temporal (5/90, 6\%) lobes. Noncodeleted tumors were most commonly centered in the frontal lobe $(35 / 58,60 \%)$, followed by the temporal lobe $(9 / 58,16 \%)$, insula $(7 / 58,12 \%)$, and parietal lobe $(5 / 58$; $9 \%)$. A statistically significant difference in the anatomic distribution of the tumors was noted $(P=.02)$; tumors of the frontal and parietal lobes were more likely to be $1 \mathrm{p} / 19 \mathrm{q}$ codeleted oligodendrogliomas, which represented 66/101 (65\%) and 15/20 (75\%) tumors in these locations, respectively. Insular and temporal tumors were more likely to be noncodeleted.

\section{Tumor Imaging Features}

Imaging characteristics of the tumors are shown in the On-line Table. Genetically defined oligodendrogliomas were characterized by poorly circumscribed borders (83/90, 92\%). Of all tumors in this series with poorly circumscribed borders, most $(83 / 109$, $76 \%$ ) were genetically defined oligodendrogliomas. Oligodendrogliomas generally lacked sharp borders on both T1- and T2weighted images (87/90, 97\% for each) and lacked a smooth contour on T2-weighted images (86/90, 96\%). The association between indistinct borders and oligodendroglioma was highly age (OR, 1.06 for each year; 95\% CI, 1.02-1.10) and were strongly predicted by a lack of circumscribed borders (OR, 16.35; 95\% CI, $6.08-50.63)$ relative to tumors with completely or partially circumscribed margins. Although tumor location was not statistically significant overall after adjusting for age and circumscription $(P=.06)$, frontal location tumors were most likely to be codeleted, and the pair-wise comparison between frontal versus temporal was significant $(\mathrm{OR}, 7.82 ; 95 \% \mathrm{CI}, 1.66-41.67 ; P=.01)$.

\section{ADC Values}

ADC sequences were available in 65 patients (On-line Table). Tumors with lower mean ADC values were more likely to be $1 \mathrm{p} /$ $19 \mathrm{q}$ codeleted oligodendrogliomas $(P=.0005)$. Receiver operating characteristic analysis identified a mean ADC value of 1.41 $\mathrm{mm}^{2} / \mathrm{s}$ as the optimal cutoff point, with an area under the curve of 0.76 . A mean ADC value of $\leq 1.41 \mathrm{~mm}^{2} / \mathrm{s}$ was $73.7 \%$ sensitive and $74.1 \%$ specific for $1 \mathrm{p} / 19 \mathrm{q}$ codeleted oligodendroglioma (Fig 4). In the multivariable analysis, both $\mathrm{ADC}$ and tumor circumscription remained statistically significant predictors of oligodendroglioma, while neither patient age nor tumor location remained significant. The odds of oligodendroglioma increased markedly in low-ADC lesions (OR, 5.66 for a half-unit decrease in overall mean ADC; 95\% CI, 1.53-27.49; $P=.02$ ) and in noncircumscribed tumors (OR, 5.24; 95\% CI, 1.27-24.42; $P=.02$ ) (Table 4).

\section{DISCUSSION}

This large retrospective study characterizes findings from commonly obtained MR imaging sequences in genetically defined oligodendrogliomas. These analyses are essential following the WHO 2016 update on CNS tumors, which has led to the reclassification of many infiltrating gliomas. Oligodendrogliomas, now 


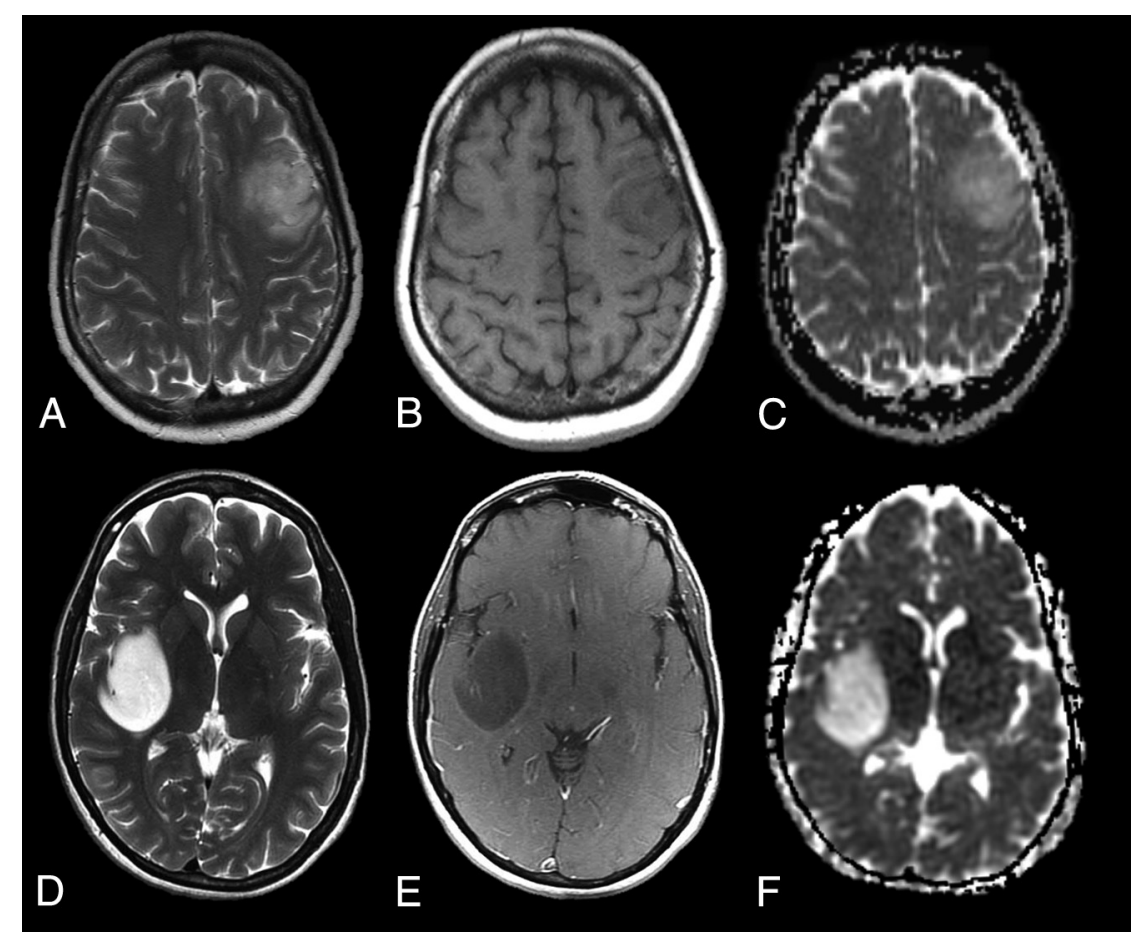

FIG 3. MR images of a prototypical genetically defined (1p/19q codeleted) oligodendroglioma (upper row) and of an astrocytoma with microscopic oligodendroglial features but no lp/19q codeletion (lower row). Axial T2-weighted ( $A$ and $D$ ), T1-weighted postcontrast ( $B$ and $E$ ), and ADC $(C$ and $F$ ) images. Genetic oligodendrogliomas tend to be located in the frontal or parietal lobe and lack circumscription, are heterogeneous, and have lower ADC values. The mean ADC value of the tumor in $C$ is $1.26 \mathrm{~mm}^{2} / \mathrm{s}$. Astrocytomas in this cohort with microscopic oligodendroglial features tend to be located in the temporal or insular lobes, are frequently well circumscribed, and have higher ADC values. The mean ADC value of the tumor shown in $F$ is $1.92 \mathrm{~mm}^{2} / \mathrm{s}$.

gliomas in our series demonstrated wellcircumscribed margins, depending on the evaluation criteria used. Other markers previously considered characteristic of oligodendrogliomas, including paramagnetic susceptibility artifacts, cystic change, and bilateral tumor burden, ${ }^{11}$ were likewise not able to distinguish genetically defined oligodendroglioma from the subset of astrocytomas with oligodendroglial morphology.

Preoperative knowledge of glioma subtype is valuable for patients and clinicians. Standard surgical treatment for glioma is maximum safe resection when possible and biopsy if resection is contraindicated, providing definitive tissue confirmation of tumor type, grade, and genetic markers such as $1 \mathrm{p} / 19 \mathrm{q}$ deletion status and IDH mutation. For patients with tumors located where complete resection may result in permanent neurologic deficits, knowledge of whether a tumor is likely to respond well to radiation and/or chemotherapy greatly impacts the risk/benefit analysis of surgery. Because 1p/19q codeletion is both prognostic of survival and predictive of response to radiochemotherapeutic treatment, it significantly informs initial

Table 3: Multivariate model including all standard anatomic imaging elements significantly associated with tumor classification as molecular oligodendroglioma in univariate analysis $^{\mathrm{a}}$

\begin{tabular}{lcccc}
\hline & Odds Ratio & $\mathbf{9 5 \%} \mathrm{Cl}$ & $\begin{array}{c}\boldsymbol{P} \\
\text { Value }\end{array}$ & $\begin{array}{c}\text { Overall } \\
\boldsymbol{P} \text { Value }\end{array}$ \\
\hline Location & & & & \\
$\quad$ Frontal & Reference & & & \\
Insular & 0.36 & $0.07-1.69$ & .20 & .06 \\
Parietal & 0.71 & $0.21-2.65$ & .59 & \\
$\quad$ Temporal & 0.13 & $0.02-0.60$ & .01 & \\
Circumscribed border & & & & \\
Partial/yes & Reference & & & \\
No & 16.35 & $6.08-50.63$ & $<.0001$ & \\
Age, 1-yr increase & 1.06 & $1.02-1.10$ & .002 & \\
\hline
\end{tabular}

${ }^{a} \mathrm{C}$ statistic $=0.84$

defined by $I D H$ mutation and $1 \mathrm{p} / 19 \mathrm{q}$ codeletion, are likely to demonstrate poorly defined tumor borders, internal heterogeneity, and a lower ADC value compared with astrocytomas with some oligodendroglial morphology. In contrast, noncodeleted infiltrating gliomas demonstrating oligodendroglial morphology, a subset of genetically defined astrocytomas, are more likely to be well-defined and internally homogeneous. Genetically defined oligodendrogliomas have a predilection for the frontal and parietal lobes.

Our results represent a shift from imaging findings previously associated with oligodendroglial tumors as defined by the prior histology-based WHO criteria. Circumscribed margins on MR imaging ${ }^{8}$ had been considered a classic appearance of oligodendroglioma, but only 3\%-8\% of genetically defined oligodendro- patient counseling and treatment planning. ${ }^{1-3}$

Efforts to correlate $1 \mathrm{p} / 19 \mathrm{q}$ codeletion with imaging features began before the discovery of the $I D H$ mutation and the current WHO revision. ${ }^{12-17}$ Previous smaller retrospective studies found that $1 \mathrm{p} / 19 \mathrm{q}$ codeletion was associated with an indistinct border on $\mathrm{T} 1$ and heterogeneity on T1 and T2 images, ${ }^{13}$ and lack of $1 \mathrm{p} / 19 \mathrm{q}$ codeletion was associated with tumor homogeneity and sharp/smooth borders. ${ }^{15}$ Regarding location, $1 \mathrm{p}$ deleted or $1 \mathrm{p} /$ $19 q$ codeleted oligodendroglial tumors tend to be found in the frontal lobes and not in the temporal lobes. ${ }^{11,12,18,19}$

In the near future, it may be possible for radiologists to classify most infiltrating gliomas before an operation with a high degree of confidence. In 2016, WHO grade II and III infiltrating gliomas are each broken into 3 categories based on molecular characteristics: astrocytoma $I D H$-mutant, astrocytoma $I D H$-wild type, and oligodendroglioma $I D H$-mutant $1 \mathrm{p} / 19 \mathrm{q}$ codeleted tumors. Tumors with mutations in $I D H$ may be identified radiographically by a number of different methods. Mutations in IDH lead to elevated levels of 2-hydroxyglutarate within tumors, which can be identified by MR spectroscopy. ${ }^{20,21}$ This technique is advancing rapidly and is now in clinical use at some centers. ${ }^{22}$ Alternatively, methods such as MR perfusion and diffusion tensor imaging have been shown to correlate with $I D H$-mutation status. ${ }^{23-25}$ Provided that these techniques, particularly spectroscopy, continue to advance at their present rate, radiographic identification of $1 \mathrm{p} / 19 \mathrm{q}$ status could be all that is necessary to categorize WHO grade II and III infiltrating gliomas by imaging. 
Relatively little has been published on the use of advanced imaging techniques to identify $1 \mathrm{p} / 19 \mathrm{q}$ codeletion. While all $1 \mathrm{p} / 19 \mathrm{q}$ codeleted oligodendrogliomas are also IDH-mutant, $1 \mathrm{p} / 19 \mathrm{q}$ codeletion itself does not have any characteristic findings on MR spectroscopy. Recent publications have evaluated the associations between codeletion and methods such as MR diffusion, MR perfusion, and $\left[{ }^{18}\right]$ F-fluoroethyl-L-tyrosine PET. ${ }^{18,26-29}$ Our finding of an association between lower ADC values with $1 \mathrm{p} / 19 \mathrm{q}$ codeletion has previously been described in small studies. ${ }^{16,30}$ Further investigation will be necessary to define the optimal way of measuring ADC and to determine the physiologic correlate of ADC values in genetically defined oligodendroglioma. ${ }^{30}$

While a strength of this current study is its large number of subjects relative to previous publications, it has limitations that should be understood when applying the findings. First, the analysis cohort comprised solely infiltrating gliomas with oligodendroglial features by microscopy. As a result, while the subgroup of $1 \mathrm{p} / 19 \mathrm{q}$ codeleted tumors should be representative of most genetically defined oligodendrogliomas, the tumors lacking $1 \mathrm{p} / 19 \mathrm{q}$ codeletion represent a subset of astrocytomas with distinct imaging and pathologic features, about which further study is

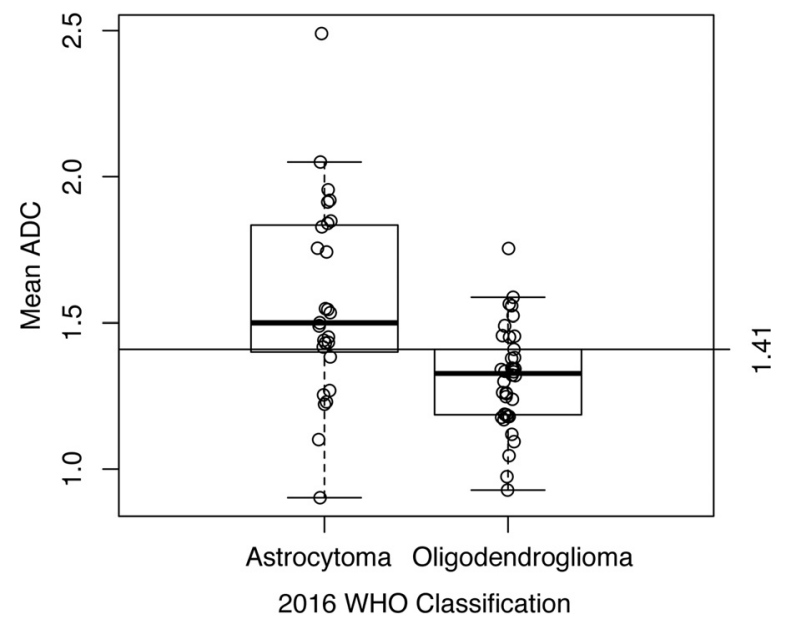

FIG 4. Boxplot demonstrating the distribution of mean ADC values by the WHO 2016 classification as oligodendroglioma or astrocytoma.

Table 4: Multivariable models of molecular oligodendroglioma status based on tumor circumscription and ADC value

\begin{tabular}{|c|c|c|c|}
\hline & Odds Ratio & $95 \% \mathrm{Cl}$ & $P$ Value \\
\hline \multicolumn{4}{|l|}{ Model A } \\
\hline \multicolumn{4}{|l|}{ Circumscribed } \\
\hline Partial/yes & Reference & & \\
\hline No & 5.24 & $1.27-24.42$ & .02 \\
\hline Overall mean ADC, 0.5 decrease & 5.66 & $1.53-27.49$ & .02 \\
\hline \multicolumn{4}{|l|}{ Model B } \\
\hline \multicolumn{4}{|l|}{ Circumscribed } \\
\hline Partial/yes & Reference & & \\
\hline No & 5.55 & $1.40-24.99$ & .02 \\
\hline Mean min ADC, 0.5 decrease & 2.75 & $0.78-11.36$ & .13 \\
\hline \multicolumn{4}{|l|}{ Model C } \\
\hline \multicolumn{4}{|l|}{ Circumscribed } \\
\hline Partial/yes & Reference & & \\
\hline No & 7.09 & $1.81-33.83$ & .007 \\
\hline Mean max ADC, 0.5 decrease & 3.89 & $1.45-12.58$ & .01 \\
\hline
\end{tabular}

warranted. Furthermore, the rare tumors that are purely astrocytic by morphology but are nonetheless IDH-mutant and 1p/19q codeleted and thus oligodendrogliomas by the 2016 criteria might exhibit different MR imaging characteristics, and further study will be needed to evaluate this question. An additional limitation is that the pretreatment images were performed at a variety of institutions during a number of years. Thus, we were unable to assess the utility of many advanced MR imaging techniques and were only able to preliminarily evaluate the association of ADC values and $1 \mathrm{p} / 19 \mathrm{q}$ codeletion. Also, because many of these patients did not have preoperative CT, our evaluation for the previous classic oligodendroglial finding of tumoral calcification was limited.

Because our evaluation of imaging features was through consensus, the interobserver variability with regard to these features remains undetermined. There was substantial and expected overlap in our judgments of tumor circumscription and border sharpness and contour, and the best of these measures to use may be the one with least intra- and interobserver variability, which could be evaluated in a future study. Last, fluorescence in situ hybridization has been and continues to be widely used to assess $1 \mathrm{p} / 19 \mathrm{q}$ codeletion, but it may occasionally result in false-positives in tumors with segmental deletions of $1 p$ and $19 q$ rather than the whole arm deletion. In the future, routine use of copy number array testing may allow more precise identification of $1 \mathrm{p} / 19 \mathrm{q}$ codeletion and thus oligodendroglioma.

\section{CONCLUSIONS}

Recent advances in the understanding of glioma pathogenesis, behavior, and treatment underscore the importance of molecular and genetic factors. As tumors become defined by their genetic makeup, as with the WHO 2016 tumor update, it is critical that imaging features that predict genetics are appreciated. Radiologists need to recognize the information already available in routine MR imaging until advanced imaging methods for the confident identification of $1 \mathrm{p} / 19 \mathrm{q}$ codeletion are routinely available. On the basis of this study, genetically defined (IDHmutant and $1 \mathrm{p} / 19 \mathrm{q}$ codeleted) oligodendroglioma is much more commonly poorly defined and infiltrative than circumscribed and is almost always heterogeneous in signal intensity. While location within the brain was not as strongly associated with tumor type, genetically defined oligodendrogliomas in this series occurred more frequently in the frontal and parietal lobes than astrocytomas.

\section{ACKNOWLEDGMENTS}

We wish to sincerely thank Dr Sonia Watson for help in manuscript preparation.

Disclosures: Derek R. Johnson—UNRELATED: Consultancy: Genentech. Caterina Giannini-RELATED: Grant: National Cancer Institute grant P50 CA108961. Robert B. Jenkins-RELATED: Grant: National Cancer Institute grant P50 CA108961. Timothy J. KaufmannUNRELATED: Consultancy: SpineThera. 


\section{REFERENCES}

1. Buckner JC, Shaw EG, Pugh SL, et al. Radiation plus procarbazine, CCNU, and vincristine in low-grade glioma. N Engl J Med 2016;374: 1344-55 CrossRef Medline

2. Cairncross JG, Wang M, Jenkins RB, et al. Benefit from procarbazine, lomustine, and vincristine in oligodendroglial tumors is associated with mutation of IDH. J Clin Oncol 2014;32:783-90 CrossRef Medline

3. van den Bent MJ, Brandes AA, Taphoorn MJ, et al. Adjuvant procarbazine, lomustine, and vincristine chemotherapy in newly diagnosed anaplastic oligodendroglioma: long-term follow-up of EORTC brain tumor group study 26951. J Clin Oncol 2013;31: 344-50 CrossRef Medline

4. Smith JS, Perry A, Borell TJ, et al. Alterations of chromosome arms $1 \mathrm{p}$ and $19 \mathrm{q}$ as predictors of survival in oligodendrogliomas, astrocytomas, and mixed oligoastrocytomas. J Clin Oncol 2000;18: 636-45 Medline

5. Lassman AB. Success at last: a molecular factor that informs treatment. Curr Oncol Rep 2013;15:47-55 CrossRef Medline

6. Louis DN, Perry A, Reifenberger G, et al. The 2016 World Health Organization Classification of Tumors of the Central Nervous System: a summary. Acta Neuropathol 2016;131:803-20 CrossRef Medline

7. Eckel-Passow JE, Lachance DH, Molinaro AM, et al. Glioma groups based on $1 \mathrm{p} / 19 \mathrm{q}$, IDH, and TERT promoter mutations in tumors. N Engl J Med 2015;372:2499-508 CrossRef Medline

8. Osborne AG. Osborne's Brain: Imaging, Pathology, and Anatomy. Salt Lake City: Amirsys; 2013:495

9. Louis DN, Ohgaki H, Wiestler OD, et al. World Health Organization Histological Classification of Tumours of the Central Nervous System. Lyon: International Agency for Research on Cancer; 2007

10. Jenkins RB, Xiao $\mathrm{Y}$, Sicotte $\mathrm{H}$, et al. A low-frequency variant at $\mathbf{8 q 2 4 . 2 1}$ is strongly associated with risk of oligodendroglial tumors and astrocytomas with IDH1 or IDH2 mutation. Nat Genet 2012;44: 1122-25 CrossRef Medline

11. Zlatescu MC, TehraniYazdi A, Sasaki H, et al. Tumor location and growth pattern correlate with genetic signature in oligodendroglial neoplasms. Cancer Res 2001;61:6713-15 Medline

12. Laigle-Donadey F, Martin-Duverneuil N, Lejeune J, et al. Correlations between molecular profile and radiologic pattern in oligodendroglial tumors. Neurology 2004;63:2360-62 CrossRef Medline

13. Megyesi JF, Kachur E, Lee DH, et al. Imaging correlates of molecular signatures in oligodendrogliomas. Clin Cancer Res 2004;10:4303-06 CrossRef Medline

14. Walker C, du Plessis DG, Fildes D, et al. Correlation of molecular genetics with molecular and morphological imaging in gliomas with an oligodendroglial component. Clin Cancer Res 2004;10: 7182-91 CrossRef Medline

15. Jenkinson MD, du Plessis DG, Smith TS, et al. Histological growth patterns and genotype in oligodendroglial tumours: correlation with MRI features. Brain 2006;129:1884-91 CrossRef Medline

16. Jenkinson MD, Smith TS, Brodbelt AR, et al. Apparent diffusion coefficients in oligodendroglial tumors characterized by genotype. J Magn Reson Imaging 2007;26:1405-12 CrossRef Medline

17. Brown R, Zlatescu M, Sijben A, et al. The use of magnetic resonance imaging to noninvasively detect genetic signatures in oligodendroglioma. Clin Cancer Res 2008;14:2357-62 CrossRef Medline

18. Kim JW, Park CK, Park SH, et al. Relationship between radiological characteristics and combined $1 \mathrm{p}$ and $19 \mathrm{q}$ deletion in World Health Organization grade III oligodendroglial tumours. J Neurol Neurosurg Psychiatry 2011;82:224-27 CrossRef Medline

19. Mueller W, Hartmann C, Hoffmann A, et al. Genetic signature of oligoastrocytomas correlates with tumor location and denotes distinct molecular subsets. Am J Pathol 2002;161:313-19 CrossRef Medline

20. Andronesi OC, Kim GS, Gerstner E, et al. Detection of 2-hydroxyglutarate in IDH-mutated glioma patients by in vivo spectralediting and 2D correlation magnetic resonance spectroscopy. $\mathrm{Sci}$ Transl Med 2012;4:116ra4 CrossRef Medline

21. Andronesi OC, Rapalino O, Gerstner E, et al. Detection of oncogenic IDH1 mutations using magnetic resonance spectroscopy of 2-hydroxyglutarate. J Clin Invest 2013;123:3659-63 CrossRef Medline

22. de la Fuente MI, Young RJ, Rubel J, et al. Integration of 2-hydroxyglutarate-proton magnetic resonance spectroscopy into clinical practice for disease monitoring in isocitrate dehydrogenase-mutant glioma. Neuro Oncol 2016;18:283-90 CrossRef Medline

23. Kickingereder P, Sahm F, Radbruch A, et al. IDH mutation status is associated with a distinct hypoxia/angiogenesis transcriptome signature which is non-invasively predictable with $\mathrm{rCBV}$ imaging in human glioma. Sci Rep 2015;5:16238 CrossRef Medline

24. Qi S, Yu L, Li H, et al. Isocitrate dehydrogenase mutation is associated with tumor location and magnetic resonance imaging characteristics in astrocytic neoplasms. Oncol Lett 2014;7:1895902 Medline

25. Xiong J, Tan W, Wen J, et al. Combination of diffusion tensor imaging and conventional MRI correlates with isocitrate dehydrogenase $1 / 2$ mutations but not $1 \mathrm{p} / 19 \mathrm{q}$ genotyping in oligodendroglial tumours. Eur Radiol 2016;26:1705-15 CrossRef Medline

26. Jansen NL, Schwartz C, Graute V, et al. Prediction of oligodendroglial histology and LOH 1p/19q using dynamic [(18)F]FET-PET imaging in intracranial WHO grade II and III gliomas. Neuro Oncol 2012;14:1473-80 CrossRef Medline

27. Chawla S, Krejza J, Vossough A, et al. Differentiation between oligodendroglioma genotypes using dynamic susceptibility contrast perfusion-weighted imaging and proton MR spectroscopy. AJNR Am J Neuroradiol 2013;34:1542-49 CrossRef Medline

28. Fellah S, Caudal D, De Paula AM, et al. Multimodal MR imaging (diffusion, perfusion, and spectroscopy): is it possible to distinguish oligodendroglial tumor grade and $1 \mathrm{p} / 19 \mathrm{q}$ codeletion in the pretherapeutic diagnosis? AJNR Am J Neuroradiol 2013;34:1326-33 CrossRef Medline

29. Saito T, Muragaki Y, Maruyama T, et al. Calcification on CT is a simple and valuable preoperative indicator of $1 \mathrm{p} / 19 \mathrm{q}$ loss of heterozygosity in supratentorial brain tumors that are suspected grade II and III gliomas. Brain Tumor Pathol 2016;33:175-82 CrossRef Medline

30. Khayal IS, Vandenberg SR, Smith KJ, et al. MRI apparent diffusion coefficient reflects histopathologic subtype, axonal disruption, and tumor fraction in diffuse-type grade II gliomas. Neuro Oncol 2011; 13:1192-201 CrossRef Medline 